\title{
COVERING FUNCTIONALS OF MINKOWSKI SUMS AND DIRECT SUMS OF CONVEX BODIES
}

\author{
SEnlin Wu, BaOfang FAn And Chan He
}

\begin{abstract}
We prove a series of inequalities concerning covering functionals of convex bodies having the form $K+L$, where $K$ is a convex body and $L$ is a segment. Several estimations of covering functionals of direct sums of convex bodies are also presented.
\end{abstract}

Mathematics subject classification (2010): 52C17, 52A20.

Keywords and phrases: Convex body, covering, Hadwiger's covering conjecture.

\section{REFERENCES}

[1] K. Bezdek, Classical Topics in Discrete Geometry, CMS Books in Mathematics/Ouvrages de Mathématiques de la SMC, Springer, New York, 2010.

[2] K. BezdeK And M.A. Khan, On the covering index of convex bodies, Aequationes Math., 90(5):879-903, 2016.

[3] K. Bezdek And Muhammad A. Khan, The geometry of homothetic covering and illumination, In Discrete Geometry and Symmetry, volume 234 of Springer Proc. Math. Stat., pages 1-30. Springer, Cham, 2018.

[4] V. Boltyanski and H. Martini, Combinatorial geometry of belt bodies, Results Math., 28(34):224-249, 1995.

[5] V. BoltyansKi AND H. MaRTini, Illumination of direct vector sums of convex bodies, Studia Sci. Math. Hungar., 44(3):367-376, 2007.

[6] V. Boltyanski, H. Martini, And P.S. Soltan, Excursions into Combinatorial Geometry, Universitext. Springer-Verlag, Berlin, 1997.

[7] P. Brass, W. Moser, AND J. PACH, Research Problems in Discrete Geometry, Springer, New York, 2005.

[8] P.M. Gruber, The space of convex bodies, In Handbook of Convex Geometry, Vol. A, B, pages 301-318. North-Holland, Amsterdam, 1993.

[9] H. MarTini, Some results and problems around zonotopes, In Intuitive Geometry (Siófok, 1985), volume 48 of Colloq. Math. Soc. János Bolyai, pages 383-418. North-Holland, Amsterdam, 1987.

[10] H. Martini AND V. Soltan, Combinatorial problems on the illumination of convex bodies, Aequationes Math., 57(2-3):121-152, 1999.

[11] V. Soltan, Affine diameters of convex bodies-a survey, Expo. Math., 23(1):47-63, 2005.

[12] V. Soltan, Lectures on Convex Sets, World Scientific Publishing Co. Pte. Ltd., Hackensack, NJ, 2015.

[13] Senlin Wu and Chan He, Covering functionals of convex polytopes, Linear Algebra Appl., 577:53-68, 2019.

[14] Senlin Wu And Ying Zhou, On the illumination of a class of convex bodies, Contributions to Discrete Mathematics, 14(1):190-202, 2019.

[15] Chuanming Zong, A quantitative program for Hadwiger's covering conjecture, Sci. China Math., 53(9):2551-2560, 2010.

[16] Chuanming Zong, Functionals on the spaces of convex bodies, Acta Math. Sin. (Engl. Ser.), 32(1):124-136, 2016. 